\title{
Los efectos de la arteterapia sobre las personas ingresadas en unidades psiquiátricas. Revisión scoping
}

\author{
The Effects of Art Therapy on Psychiatric Inpatient Units. Scoping Review
}

\section{Lucía Pérez Ruiz', Rocío Juliá-Sanchis², Marcelino Vicente Pastor-Bernabeu², Ángela Sanjuan-Quiles ${ }^{2}$}

${ }^{1}$ Grado en Enfermería.

${ }^{2}$ Departamento de Enfermería, Universidad de Alicante. Alicante, España.

Contacto: rjulia@ua.es

Fecha de recepción: 5 de marzo de 2018 / Fecha de aceptación: 4 de mayo de 2018

\begin{abstract}
Resumen
Introducción: Nuevas estrategias arteterapéuticas inclusivas centradas en la persona y no en su etiqueta diagnóstica se están abriendo paso en las unidades de hospitalización psiquiátrica como coadyuvantes a los métodos habituales de psicoterapia y psicofármacos. Esta implementación viene dada por los reputados beneficios reportados en contextos comunitarios.

Objetivo: Conocer los resultados de las intervenciones de arteterapia aplicada en unidades de hospitalización psiquiátrica de adultos.

Metodología: Revisión en las bases de datos electrónicas CINAHL, MEDLINE, PsycINFO, SCOPUS y WOS.

Resultados: Se identificaron 1101 registros, de los que 1081 fueron excluidos en base a la información incluida en su título y resumen. 21 artículos fueron analizados para la revisión, de los cuales 14 cumplieron los criterios de inclusión/exclusión. Las intervenciones se clasificaron en 4 categorías: musicoterapia, terapias creativas artesanales (dibujo, arcilla y marmolado), terapias sensoriales y expresión corporal.

Conclusiones: La estimulación de la expresión emocional es un recurso esencial para que los pacientes alcancen el máximo nivel de salud y calidad de vida que su condición les permita. La implementación de intervenciones de arteterapia en unidades de hospitalización psiquiátrica tiene efectos beneficiosos en las personas con trastorno mental grave. Aunque son escasos los estudios encontrados, todos coinciden en destacar el valor positivo en el bienestar físico y mental de los participantes gracias a la expresión de emociones, la reducción de sintomatología negativa, a los procesos de adaptación al trastorno y la adherencia al tratamiento.
\end{abstract}

Palabras clave: arteterapia, salud mental, hospital

\begin{abstract}
Introduction: New inclusive therapeutic art strategies focused on the person and not on their diagnostic label are making their way into the psychiatric hospitalization units as coadjutants to the usual methods of psychotherapy and psychotropic drugs due to the renowned benefits reported in community contexts.

Objective: To know the results of art therapy interventions applied in adult psychiatric hospitalization units. Methodology: Electronic databases CINAHL, MEDLINE, PsycINFO, SCOPUS and WOS were reviewed.

Results: 1101 papers were identified, of which 1081 were excluded based on the information included in their title and abstract. 21 articles were analyzed of which 14 met the inclusion/exclusion criteria. The interventions were classified into 4 categories: music therapy, creative craft therapies (drawing, clay and marble), sensory therapies and body language.

Conclusions: The stimulation of expression is an essential resource for patients to acquire new strategies to reach the highest level of health and quality of life that their condition allows them. The implementation of art therapy interventions in psychiatric hospitalization units has beneficial effects in people with severe mental disorder. Although few studies were found, all agree in highlighting the positive value in the physical and mental wellbeing of the participants due to the expression of emotions, the reduction of negative symptomatology, to the adaptation processes to the disorder and the adherence to the treatment.
\end{abstract}

Keywords: art therapy, mental health, hospital. 


\section{Introducción}

Los trastornos mentales graves se caracterizan por ser una combinación de alteraciones del pensamiento, percepción, emociones, conducta y relaciones con los demás (OMS, 2017). Estas alteraciones pueden manifestarse de manera distinta en personas con la misma etiqueta diagnóstica, dando lugar a procesos concretos e individuales.

A pesar de la diversidad de técnicas psicoterapéuticas y del desarrollo de nuevos psicofármacos, muchas personas con problemas de salud mental no obtienen los resultados esperados debido a que, en la mayoría de los casos, se requiere de un periodo mínimo de tiempo para apreciar sus efectos beneficiosos o, por lo contrario, percibir que los síntomas persisten a pesar de la aplicación de estas terapias tradicionales (Ruiz, Aceituno y Rada, 2017). Como consecuencia, se están abriendo paso nuevas estrategias terapéuticas centradas en la persona en las unidades de hospitalización psiquiátrica como coadyuvantes a los métodos habituales (Crone et al., 2012) como por ejemplo la arteterapia.

La arteterapia es una intervención psicoterapéutica alternativa compuesta por diversas actividades que facilitan la comunicación y la expresión de sentimientos. (British Association of Art Therapists, 2016). Esta terapia ofrece la oportunidad de conectar el mundo interno con el externo, es decir, ofrece al individuo interactuar con el entorno en el momento en que, por su deterioro cognitivo y funcional, no posee la capacidad de expresar lo que siente o bien por la gran carga emocional que estos pensamientos poseen (Halužan, 2012; Osborn y Tate, 2011).

Esta práctica terapéutica no busca tratar la patología con su sintomatología asociada, sino que busca integrar a la persona con sus aspectos emocionales y espirituales, promoviendo cambios en las áreas afectiva, interpersonal y relacional, empoderándola desde un punto de vista más humano (Consellería de Sanitat, 2014; Crone et al., 2012; Shorters, 2011) ya que "El arte humaniza los espacios" y permite una mejor calidad de vida(Ventura et al. 2011).

La música, la danza o la pintura son algunos ejemplos de arteterapia. Todas ellas han sido consideradas beneficiosas desde tiempos ancestrales; no obstante, su uso terapéutico comenzó a partir de la Segunda Guerra Mundial, cuando se desarrollaron las primeras investi- gaciones que demostraban sus ventajas, popularizándose en Estados Unidos, Gran Bretaña, Japón, Alemania o Francia. No obstante, en países del sur de Europa, como España, todavía es necesario conocer, desarrollar e implementar estas intervenciones, ya que su incorporación en centros sanitarios y sociosanitarios está siendo muy tímida (Ventura et al., 2011), y la enfermería podría ser el colectivo profesional que liderara este proceso.

El propósito principal de este trabajo fue conocer los resultados de las intervenciones de arteterapia aplicada en unidades de hospitalización psiquiátrica de adultos. De manera secundaria nos propusimos recopilar, agrupar y describir los resultados de los trabajos recopilados.

\section{Metodología}

Llevamos a cabo una revisión de alcance o scoping review (Armstrong, Hall, Doyle y Waters, 2011) como estrategia de mapeo de la literatura existente. Los criterios de elección fueron:

1. Artículos originales y revisiones.

2. Estudios publicados en inglés o español.

3 . Estudios que emplearan intervenciones de arteterapia.

4. Estudios cuya muestra fueran personas con trastorno mental grave de origen no orgánico.

5. Estudios cuyo procedimiento se llevara a cabo en unidades de hospitalización psiquiátrica.

6. Estudios publicados de acceso abierto.

Los criterios de exclusión fueron personas con trastorno mental grave menores de 15 años o que la intervención se realizara en un contexto comunitario.

La estrategia de búsqueda fue diseñada para recuperar el mayor número de referencias. Las autoras LPR y RJS emplearon la misma estrategia en las bases de datos electrónicas CINAHL, MEDLINE, PsycINFO, SCOPUS y Web of Science, desde 2 de febrero hasta 31 de marzo de 2017 usando los siguientes términos: "art therapy" AND ("mental illness" OR "psychiatric" OR "psychiatry" AND "hospital").

\section{Selección de estudios y recopilación de datos}

La selección de los registros fue llevada a cabo de manera independiente tras la lectura de los títulos y resúmenes. Posteriormente se procedió a la lectura del 
texto completo, encontrando textos dudosos. En esos casos, se decidió tras la consulta con la tercera investigadora (ASQ).

Posteriormente, LPR extrajó en cuadros de evidencia la información relevante de los estudios incluidos y un segundo autor (RJS) verificó todos los datos extraí- dos para obtener la siguiente información: estudio (autores y año de publicación), tipo de estudio, características de la muestra (tamaño, datos demográficos), características de la intervención y resultados obtenidos. Los desacuerdos se resolvieron mediante discusión entre las autoras.

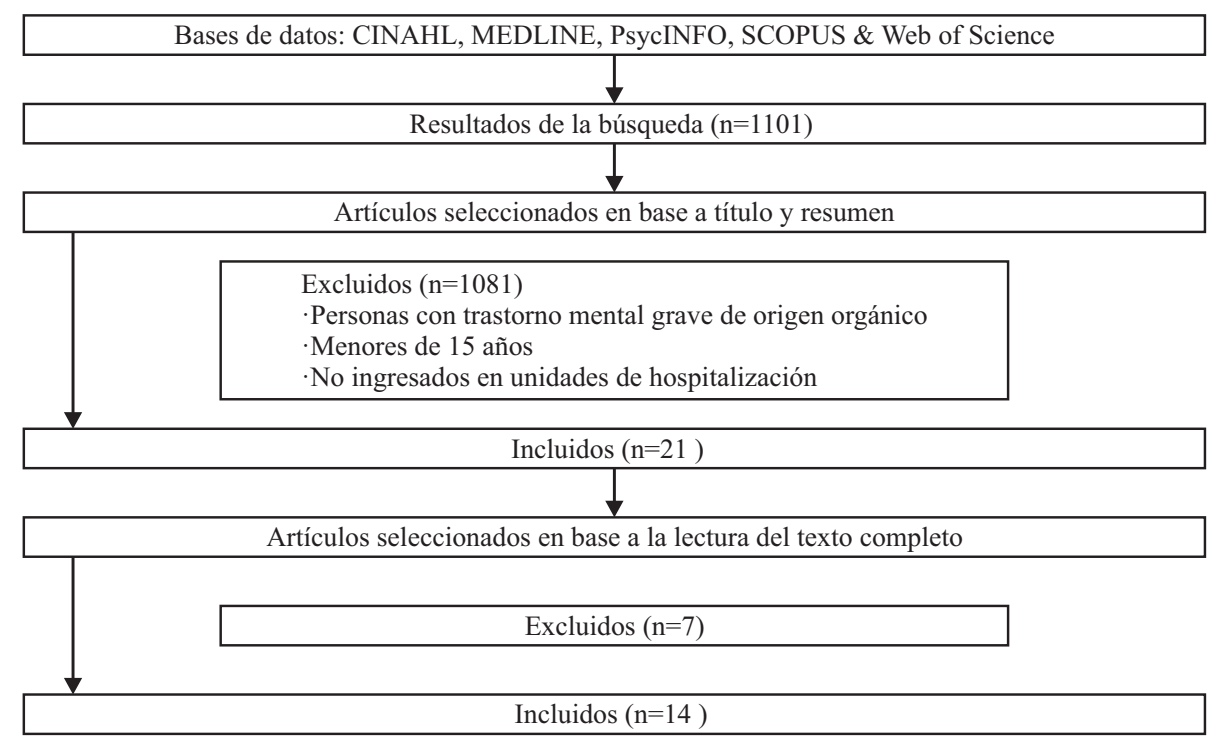

Figura 1: Identificación y selección de estudios para la revisión scoping

\section{Resultados}

Usando la estrategia de búsqueda, 1101 registros fueron encontrados, de los cuales 1081 fueron excluidos en base a la información incluida en su título y resumen (ver figura 1). Tras eliminar los artículos repetidos, 21 fueron identificados, recuperados y analizados. Tras la lectura completa del texto, finalmente fueron 14 los artículos seleccionados.

Debemos destacar que fueron muchos los artículos eliminados por no estar relacionados con el tema, ya que ART también se utiliza como el acrónimo de antiretroviral therapy. Por ese motivo se encontraron gran cantidad de artículos que posteriormente no se incluyeron en la revisión.

\section{Artículos seleccionados}

Respecto al diseño de los estudios, siete presentaron un diseño cuantitativo, de los que tres fueron casos y controles. Seis estudios tuvieron diseño cualitativo y uno fue una revisión de la literatura. Respecto a la información de la muestra, el número de participantes estuvo entre 2-403. Entre el 37 y el $100 \%$ de las participantes fueron mujeres. El rango de edad se situó entre 18-65 años (ver Tabla 1).

\section{Descripción de las intervenciones}

Las intervenciones se agruparon en 4 grandes grupos: musicoterapia, terapias creativas artesanales (dibujo, arcilla y marmolado), terapias sensoriales y expresión corporal.

El tiempo de duración de las intervenciones fue variable, situándose entre las seis semanas y un año. Las sesiones de 60-90 minutos (ver Tabla 1).

Entre los profesionales que dirigieron la actividades, se encontraron: enfermeras/os especialistas o generalistas $(n=4)$, médicos/as $(n=3)$, un terapeuta ocupacional $(n=1)$ y terapeutas $(n=4)$; en dos estudios fue interdisciplinar. 
La intervención más empleada fue la musicoterapia con el propósito de aumentar la motivación en trastornos como esquizofrenia, depresión, trastorno bipolar y trastornos psicosomáticos.

Por ejemplo, Gold et al. (2013) emplearon la música como complemento al tratamiento convencional, obteniendo resultados significativos de mejora en la motivación hacia esta y otras terapias, así como en síntomas negativos, la funcionalidad, la vitalidad, y el estado general de sus participantes. Además, obtuvieron resultados muy prometedores sobre la interacción social.

Ross et al. (2008) se centraron en personas con trastorno dual, quienes mostraron gran interés por asistir a las terapias musicales y refirieron haber reducido su malestar psíquico y mejorado su funcionamiento general (control de síntomas, control de impulsos, motivación para el cambio) y su relación con los demás.

Por su parte, Curreri (2013) empleó la musicoterapia creada al azar y desorganizada como herramienta para mejorar la rigidez mental de los pacientes. Los participantes valoraron la intervención como una experiencia positiva y una buena herramienta de apoyo para mejorar su estado de salud.

No obstante, la música tiene más usos, ya que también puede emplearse para la expresión corporal mediante la danza. Dentro de este grupo de intervenciones, encontramos el taichí. Con su revisión, Wang et al. (2014) concluyeron que el taichí es beneficioso para reducir la ansiedad, la depresión y aumentar la calidad de vida. Sugirieron la necesidad de mejorar el conocimiento sobre los efectos de este ejercicio en el bienestar psicológico, ya que a nivel físico ya habían sido ampliamente estudiados.

Otro grupo de intervenciones fueron las relacionadas con la terapia creativa artesanal, donde se incluyeron el dibujo, la pintura u otras intervenciones creativas. Entre los beneficios extraídos estuvieron el incremento de la motivación (Hung \& Ku, 2015), la reducción significativa de los síntomas positivos y negativos en pacientes con esquizofrenia (Montag et al., 2014; Utaş Akhan \& Atasoy, 2017), la reducción de los niveles de ansiedad de pacientes con trastorno bipolar (Utaş Akhan \& Atasoy, 2017), el aumento de la conciencia emocional y de la capacidad de reflexión sobre los estados mentales emocionales de los demás (de Morais et al., 2014).
El trabajo de George y Kasinathan (2015) incluyó una muestra particular de jóvenes con problemas legales, de conducta y de salud mental. Su intervención se centró en el diseño y ejecución de un mural con el que se consiguió infundir entre los participantes sentido de logro, empoderamiento, trabajo en equipo, participación, cohesión grupal y posesión acumulada del propio mural y del espacio que ocupaba. Todos los participantes reconocieron cambios ambientales positivos con el mural en el patio de la unidad.

Al igual que Montag et al. (2014), esta actividad no estuvo dirigida, permitiendo a los participantes crear lo que quisieran y utilizar cualquier herramienta disponible: mesas de trabajo, materiales artísticos como agua y pintura para carteles, lápices, lápices de colores, marcadores, crayones, pasteles, papel y pinceles de diferentes tamaños, tableros de dibujo, arcilla, herramientas, un caballete e instalaciones de almacenamiento.

Siguiendo con las terapias creativas, la terapia con arcilla demostró ser beneficiosa en personas con trastorno mental grave al promover la liberación de emociones, la creatividad, la conciencia de uno mismo, así como la percepción de autocontrol de las emociones a través de sus manos (de Morais, Roecker, Jodas y Jacklin, 2014b). Se observó una reducción de los síntomas negativos afectivos y emocionales asociados a la enfermedad, como la apatía, síntomas depresivos y ansiedad, estrés, miedo agitación, angustia, agresión, retraimiento social. A estas ventajas psicológicas se sumaron los beneficios físicos como la buena postura, la estabilidad y el equilibrio.

En línea con los resultados anteriores, Caddy, Crawford, y Page (2012) además apuntaron a la mejora significativa de la autoestima, la autoeficacia, la autonomía y de la interacción social. Teglbjaerg (2011) complementa estos resultados con su estudio comparativo entre personas con trastornos psicóticos (esquizofrenia severa) y no psicóticos (depresión, ansiedad y trastornos de personalidad) y los sus efectos a corto y largo plazo, afirmando que ambos grupos reportaron efectos positivos en los dos momentos de medición, la adquisición de estrategias para afrontar los problemas diarios.

El último grupo de intervenciones fueron las terapias sensoriales gracias a los trabajos de Ingeberg, Wikstrøm y Berg (2012) y Nanda, Eisen, Zadeh, y Owen (2011) quienes emplearon el arte visual. Estos 
últimos expusieron a los pacientes ingresados en una unidad a imágenes de naturaleza realista, a arte abstracto o a nada, siendo la primera expresión artística la que consiguió mejores puntuaciones en los niveles de ansiedad y agitación de los ingresados. Pero con este trabajo intentan ir más allá del beneficio para el paciente e incluyen además las ventajas económicas de implementar este tipo de intervención, ya que, según defiende, mediante imágenes de naturaleza realista, se reduce la necesidad de los pacientes de medicación a demanda para la agitación y la ansiedad. De esta manera, se puede ahorrar costes sanitarios de medicación, tiempo de personal y de farmacia.

Otro ejemplo de trabajo relacionado con las terapias sensoriales es el realizado por Knight, Adkison y Kovach, (2010), quienes estimulan no solo la visión sino también el olfato, el oído, el tacto y el gusto, con el propósito de reducir los síntomas con estrategias alternativas y naturales. Para ello emplearon aromas, dulces, música, sonidos relajantes, estimulación táctil, imágenes en las paredes, lámparas de lava...; aunque son los aceites esenciales inhalados los que han demostrado mejorar la relajación. La mayor ventaja de este trabajo fue que no necesita habilidades técnicas específicas para llevarla a cabo, adaptándose a aquellos individuos con deterioro cognitivo o de memoria. Para las personas con trastornos psiquiátricos, representan una terapia sencilla de realizar, representa una distracción y posee efecto calmante en personas con ansiedad, depresión o confusión. Son beneficiosas también para los trabajadores del centro, ya que disfrutan igualmente mientras acompañan al participante. Un punto clave de esta intervención es el hecho de que los participantes eligen libremente su terapia entre diversas posibilidades según sus preferencias. Sin embargo, a pesar de las notables ventajas, no hubo una diferencia significativa entre el grupo control de terapia tradicionales y el grupo de terapia multisensorial; ambos reportaron beneficios en los síntomas psiquiátricos después de las intervenciones. No obstante, el estudio concluye que es una buena opción de tratamiento, por ser más humanizada, individualizada e integradora para controlar los síntomas y por el beneficio de empoderar a la persona para elegir su tratamiento.

\section{Discusión}

En esta revisión de alcance, se incluyeron 14 estudios que evaluaron el impacto de las intervenciones de arte terapia en entornos de salud mental hospitalarios.
Las intervenciones se clasificaron en 4 grandes grupos: musicoterapia, terapias creativas artesanales, terapias sensoriales y expresión corporal.

El arte y la ciencia en enfermería han caminado separados durante mucho tiempo (Ingeberg et al., 2012), aunque, a la vista de los resultados, los profesionales de enfermería actualmente desarrollan actividades arteterapéuticas con resultados óptimos tanto para la salud física como para la salud mental de las personas ingresadas en unidades de hospitalización, siguiendo así el modelo de Florence Nightingale, quien identificó la expresión artística como una importante parte de la atención enfermera en el paciente hospitalizado.

Los estudios en esta revisión demostraron que la arteterapia en unidades de hospitalización psiquiátrica tiene efectos beneficiosos para personas con trastorno mental grave en una gran variedad de medidas de bienestar psicológico, que incluyen depresión, ansiedad, manejo general del estrés, autoestima e interacción social. Sin embargo, no está claro si es una intervención aceptable, ya que la certeza de la evidencia existente es baja (Ruiz et al., 2017). Entre los factores relacionados con la baja fiabilidad de las fuentes se encuentran: 1) la heterogeneidad diagnóstica de las muestras, lo que impide conocer qué intervención se adapta mejor a según qué etiqueta diagnóstica; 2) las restricciones metodológicas de la mayoría de los estudios, como el uso de muestras reducidas, diseños con escaso control de sesgos, etc. (administración de medicación simultánea a la intervención, la inclusión de los gustos personales de los participantes para seleccionar la intervención a realizar), con lo que se desconoce si los beneficios son por la propia terapia, por la relación con el terapeuta, por el tratamiento tradicional que se ha mantenido o una combinación de todas; 3) la naturaleza descriptiva y naturalista de algunos estudios no aportó evidencia medible para confirmar los beneficios que finalmente los participantes reportan.

Ruiz et al. (2017) plantean la dificultad de determinar el balance riesgo/beneficio de participar en este tipo de terapias, debido a la baja certeza de la evidencia sobre la efectividad de las mismas. Sin embargo, las conclusiones de la mayoría de trabajos plantean que, aunque los resultados pueden no poseer altos estándares de calidad metodológica, los riesgos para los participantes son ínfimos o inexistentes y por el contrario sus efectos sobre los síntomas y sobre la humanización de los cuidados es incuestionable. Además, ofrecen la 
oportunidad de realizar actividades recreativas y placenteras que incrementan la motivación de las personas con que las realizan, mejorando la adherencia a los tratamientos tradicionales, la satisfacción con la asistencia recibida y con ellos mismos (Solli et al., 2013; Osborn \& Tate, 2011) y así reducir o sustituir la medicación, haciendo a los pacientes menos dependientes de estas (Shorters, 2011) y empoderándolos para que sean capaces de controlar sus síntomas con técnicas sencillas pero valiosas (Knight et al., 2010).

Teniendo en cuenta estas consideraciones, es conveniente realizar más estudios con un diseño de investigación riguroso, para establecer la eficacia de la arteterapia en la mejora del bienestar psicológico y su potencial para ser utilizada en intervenciones para poblaciones con diversas condiciones clínicas, con los sesgos mínimos y teniendo en cuenta los factores positivos de humanización que proporcionan.

Al analizar los hallazgos, tras agrupar las intervenciones arteterapéuticas, encontramos que las intervenciones creativas artesanales poseían la capacidad de promover la conciencia emocional, la liberación de emociones y el autocontrol de las mismas, y favorecer un espacio donde reflexionar sobre los estados mentales emocionales de los demás (de Morais et al., 2014), además de mejorar la creatividad, la autoestima, la auto eficacia, la autonomía, la comunicación y la interacción social. Específicamente fueron ventajosas para reducir la ansiedad en personas con trastorno bipolar (Utaş Akhan \& Atasoy, 2017), para reducir los síntomas positivos y negativos en personas con esquizofrenia, como la apatía, síntomas depresivos y ansiedad, estrés, miedo agitación, angustia, agresión y retraimiento social (Utaş Akhan \& Atasoy, 2017). Sin embargo, debemos remarcar los resultados discordantes de Montag et al. (2014) respecto a los efectos de la intervención creativa artesanal en personas con depresión, ya que en su estudio no se obtuvieron cambios significativos. Crear, disfrutar, desconectar y relajarse de manera no directiva fue el propósito de todas los trabajos; es decir, que los participantes pudiesen elegir crear lo que querían hacer y cómo lo querían hacer.

A pesar de que el arte visual ha resultado ser una buena herramienta para mejorar la comunicación del paciente y el manejo de síntomas de ansiedad y agitación, el efecto del arte abstracto fue contradictorio. Ingeberg et al. (2012) defendieron que tanto el arte figurativo como el no figurativo favorecía la comunicación profesional-paciente, pero Nanda et al. (2011) consideraron que el arte abstracto producía efectos negativos.

La música ha demostrado tener efectos beneficiosos, como relajar, motivar, divertir, reducir el dolor o la ansiedad (Shorters, 2011), indiferentemente del género, edad, cultura o idioma de los participantes. Las intervenciones de musicoterapia recogidas en esta revisión tuvieron un enfoque integrador, donde primaba la persona y la búsqueda del mejor manejo de su sintomatología, fortaleciéndola, empoderándola y apoyándola (Gold et al., 2013; Solli, Rolvsjord y Borg, 2013). Con las intervenciones se redujeron los síntomas negativos que afectan a la capacidad social básica de las personas con trastorno psicótico (Gold et al., 2013), mejoró la sintomatología de las personas que padecían trastornos depresivos y aumentó la adherencia al tratamiento, siendo una herramienta potencial combinada o no con las medidas farmacológicas y psicosociales (Brandes et al., 2010). En línea con nuestros hallazgos, aunque en contextos diferentes, como en la atención en personas con trastornos mentales en la comunidad (Crone et al., 2013), personas con demencia (Staal et al., 2007) o en personas en proceso oncológico activo (Bradt, Dileo, Magill, y Teague, 2016), la música obtuvo similares efectos favoreciendo al bienestar psicológico, aliviando la ansiedad, la agitación, la depresión, el dolor, la fatiga y la calidad de vida asociadas a sus procesos.

\section{Limitaciones de estudio}

Dada la metodología establecida, el número de trabajos revisados ha sido bajo. Sin embargo, la revisión de alcance o scoping review puede priorizar los estudios que estén más fácilmente disponibles, siendo sólo para la realización de una revisión sistemática necesario localizar todos los estudios sobre el tema a estudio de manera estricta (Armstrong, 2011).

A pesar de que este estudio puede ser considerado como una contribución a la base de conocimiento de la terapia artística, debemos seguir recopilando la nueva evidencia con el fin de obtener una comprensión más profunda de los factores terapéuticos de las intervenciones descritas, así como programas para evaluar la eficiencia y la eficacia.

\section{Conclusión}

Los hallazgos de nuestra revisión muestran que la implementación de intervenciones de arteterapia en 
unidades de hospitalización psiquiátrica tienen efectos beneficiosos para personas con trastorno mental grave.

A pesar de que son escasos los estudios que demuestran de manera fiable sus beneficios, todos coinciden en destacar el valor positivo en el bienestar físico y mental de los participantes gracias a la expresión de emociones, la reducción de sintomatología negativa, a los procesos de adaptación al trastorno y la adherencia al tratamiento. Para muchos participantes, las actividades terapéuticas de arte fueron tan importantes y tan útiles como los tratamientos tradicionales.

La estimulación de la expresión es un recurso esencial para que los pacientes adquieran nuevos recursos para alcanzar el máximo nivel de salud y calidad de vida que su condición les permita. Por ello la arteterapia debe ser implementada en los recursos asistenciales de salud mental, siendo los profesionales de enfermería quienes lo lideren.

\section{Implicaciones para la investigación futura y la práctica clínica}

Los hallazgos confirman el valor y los beneficios de las intervenciones artísticas en las unidades de hospitalización. Como tal, se suma a la base actual de pruebas en desarrollo sobre el uso del arte y la creatividad en la recuperación y rehabilitación de la salud mental. Futuras investigaciones podrían incluir un diseño mixto longitudinal con mayor muestra y menos sesgos, para cuantificar y explorar la mejoría en el cambio en diferentes medidas de resultados al final de la intervención, como las obtenidas en esta revisión. Además, sigue siendo necesario investigar la relación costo-efectividad de las intervenciones, la aceptación y los niveles de cumplimiento de los participantes con diferentes etiquetas diagnósticas y el impacto del tipo de arte en los resultados.

\section{Bibliografía}

1. Armstrong, R., Hall, B.J., Doyle, J., \&Waters, E. (2011) Cochrane Update. 'Scoping the scope' of a cochrane review. Journal of Public Health. 33 (1) 147-150 doi:10.1093/pubmed/fdr015

2. Bradt, J., Dileo, C., Magill, L., \& Teague, A. (2016) Music interventions for improving psychological and physical outcomes in cancer patients. The Cochrane Library.

3. Brandes, V., Terris, D. D., Fischer, C., Loerbroks, A., Jarczok, M.N., Ottowitz, G. \& Thayer, J.F. (2010). Receptive music therapy for the treatment of depression: a proof-of-concept study and prospective controlled clinical trial of efficacy. Psychotherapy and psychosomatics. 79(5), 321-322.

4. British Association of Art Therapists [BAAT]. http://www.baat.org/about-Art-Therapy Accessed: 22 February
2018

5. Caddy, L., Crawford, F., \& Page, A.C. (2012). Painting a path to wellness': correlations between participating in a creative activity group and improved measured mental health outcome. Journal of psychiatric and mental health nursing, 19(4), 327-333.

6. Consellería de Sanitat (2014) Estrategia para la atención a pacientes crónicos en la Comunidad Valenciana. http://publicaciones.san.gva.es/publicaciones/documentos/V. 2792-2014.pdf. Ed. Generalitat. Consellería de Sanitat. Accessed: 26 February 2018)

7. Crone, D.M., O'Connell, E.E., Tyson, P.J., Clark-Stone, F., Opher S. \& James D.V.B. (2012) It helps me make sense of the world': the role of an art intervention for promoting health and wellbeing in primary care: perspectives of patients, health professionals and artists. Journal of Public Health, 20(5), 519-524.

8. Crone, D.M., O'Connell, E.E., Tyson, P.J., Clark-Stone, F., Opher S. \& James D.V.B. (2013) Art Lift' intervention to improve mental wellbeing: An observational study from UK general practice. International Journal of Mental Health Nursing. 22, 279-286. doi: 10.1111/j.1447-0349.2012.00862.x

9. Curreri, E. (2013). Aesthetic perturbation: Using a chance/aleatoric music therapy intervention to reduce rigidity in adult patients with psychiatric disorders. Music Therapy Perspectives, 31(2), 105-111.

10. de Morais, A.H., Nazario Dalécio, M.A., Vizmann, S., de Carvalho Bueno, V.L.R., Roecker, S., Jodas Salvagioni, D.A et al. (2014a) Effect on scores of depression and anxiety in psychiatric patients after clay work in a day hospital. The Arts in Psychotherapy, 41(2), 205-210.

11. de Morais, A. H., Roecker, S., Jodas Salvagioni, D.A. \& Jacklin Eler, G. (2014b). Significance of clay art therapy for psychiatric patients admitted in a day hospital. Investigación y Educación en Enfermería, 32(1), 128-138.

12. George O. y Kasinathan, J. (2015) Mural art therapy for young offenders hospitalised with a mental illness. Australasian Psychiatry 23(1)

13. Gold, C., Mössler, K., Grocke, D., Heldal, T.O., Tjemsland, L., Aarre, T., et al. (2013). Individual music therapy for mental health care clients with low therapy motivation: multicentre randomised controlled trial. Psychotherapy and psychosomatics, 82(5), 319331.

14. Halužan, M (2012). Art therapy in the treatment of alcoholics. Alcoholism and psychiatry research, 48(2), 99-105.

15. Hung, C.C., \& Ku, Y.W. (2015). Influencing and moderating factors analyzed in the group art therapy of two schizophrenic inpatients. BioMedicine, 5(4).

16. Ingeberg, M.H., Wikstrøm, B.M., \& Berg, A. (2012). The essential dialogue: a Norwegian study of art communication in mental health care. Journal of psychosocial nursing and mental health services, 50(8), 22-30.

17. Knight, M., Adkison, L., \& Kovach, J.S. (2010). A comparison of multisensory and traditional interventions on inpatient psychiatry and geriatric neuropsychiatry units. Journal of psychosocial nursing and mental health services, 48(1), 24-31.

18. Montag, C., Haase, L., Seidel, D., Bayerl, M., Gallinat, J.,Herrmann, U., \& Dannecker, K. (2014). A pilot RCT of psychodynamic group art therapy for patients in acute psychotic episodes: feasibility, impact on symptoms and mentalising capacity. PloS one, 9(11), e112348.

19. Nanda, U., Eisen, S., Zadeh, R.S., \& Owen, D. (2011) Effect of visual art on patient anxiety and agitation in a mental health facility and implications for the business case. Journal of psychiatric and mental health nursing, 18(5), 386-393.

20. Osborn, L.A., \& Tate, F.B. (2011). Using Art to Empower Clients During a Facility Move. Journal of psychosocial nursing and mental health services, 49(6), 32-37.

21. OMS, Organización Mundial de la Salud (Abril 2017). Mental disorders. 
http://www.who.int/mediacentre/factsheets/fs396/es/. Accessed: 22 April 2017

22. Ross, S., Cidambi, I., Dermatis, H., Weinstein, J., Ziedonis, D., Roth, S., et al. (2008). Music therapy: a novel motivational approach for dually diagnosed patients. Journal of Addictive Diseases, 27(1), 41-53. http://dx.doi.org/10.1300/J069v27n01_05

23. Ruiz, MI., Aceituno, D., \& Rada G. (2017) Arteterapia, ¿es efectiva en Ia esquizofrenia? Medwave. 17(Suppl1):e6845 doi: 10.5867/medwave.2017.6845

24. Shorters, V. (2011). Creating a hospital-based arts project for older people: Older adults using mental health services can develop a sense of accomplishment by taking part in arts-based therapeutic activities. Mental Health Practice, 15(3), 24-26.

25. Solli, H.P., Rolvsjord, R., \& Borg, M. (2013). Toward understanding music therapy as a recovery-oriented practice within mental health care: A meta-synthesis of service users' experiences. Journal of Music Therapy, 50(4), 244-273.

26. Staal, J.A., Matheis, R., Collier, L., Calia, T., Hanif, H., \& Kofman, E.S. (2007). The effects of Snoezelen (multi-sensory behavior therapy) and psychiatric care on agitation, apathy, and activities of daily living in dementia patients on a short term geriatric psychiatric inpatient unit. The International Journal of Psychiatry in Medicine, 37(4), 357-370.

27. Teglbjaerg, H.S. (2011). Art therapy may reduce psychopathology in schizophrenia by strengthening the patients' sense of self: a qualitative extended case report. Psychopathology, 44(5), 314318. DOI: 10.1159/000325025

28. Utaş Akhan, L., \& Atasoy, N. (2017). Impact of marbling art therapy activities on the anxiety levels of psychiatric patients. Journal of H uman Sciences, 14 ( 2), 2121 - 2128 . doi:10.14687/jhs.v14i2.4397

29. Ventura Bordes, A., Giménez Sierra, Á., Sala Lopez, R., Climent Suñer, M.Á., Moreno Cotarelo, A., Valdivia Pérez, A. et al. (2011). CuidArt: Art Project of the Health Department of Dénia. Arte, I n d i viduo y Soc i e d a d. 23, $165-180$. http://dx.doi.org/10.5209/rev_ARIS.2011.v23.36753

30. Wang, F., Lee, E.K.O. Wu, T., Benson, H., Fricchione, G., Wang, W., et al. (2014) The effects of tai chi on depression, anxiety, and psychological well- being: a systematic review and metaanalysis. International journal of behavioral medicine, 21(4), 605617. DOI 10.1007/s12529-013-9351-9 


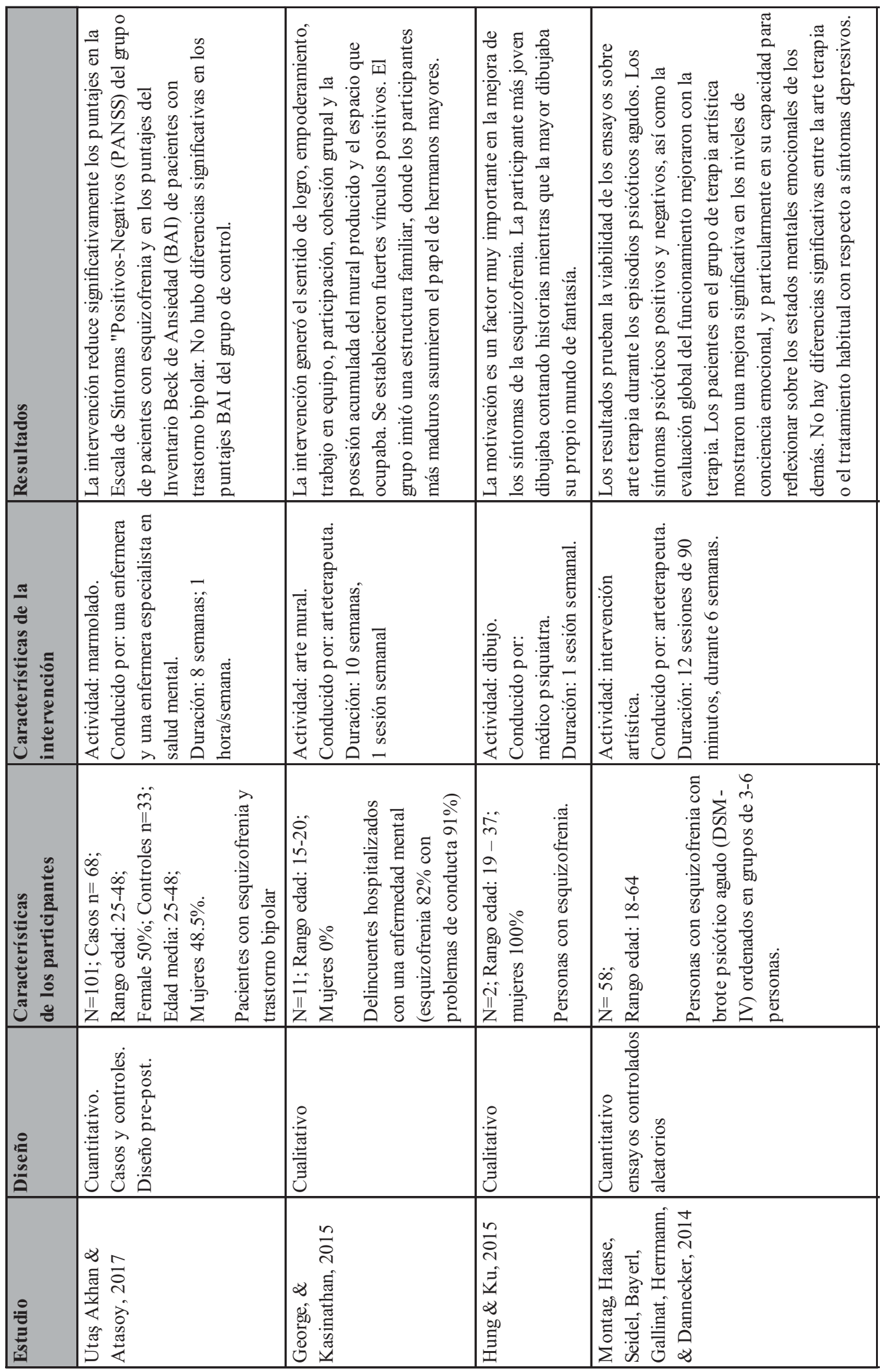




\begin{tabular}{|c|c|c|c|c|c|}
\hline 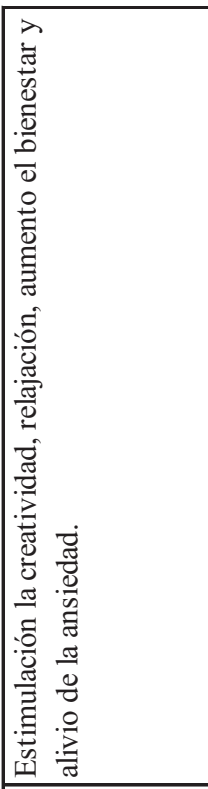 & 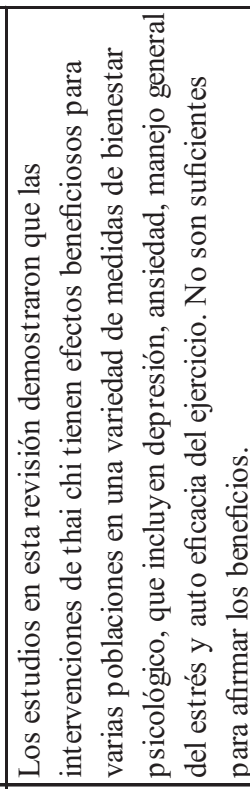 & 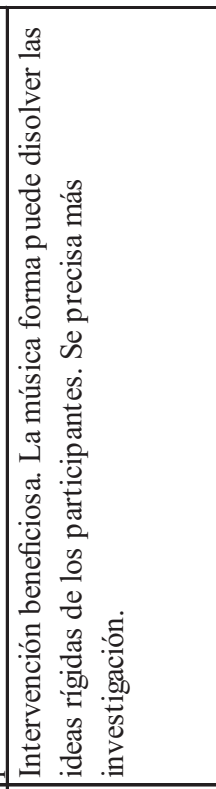 & 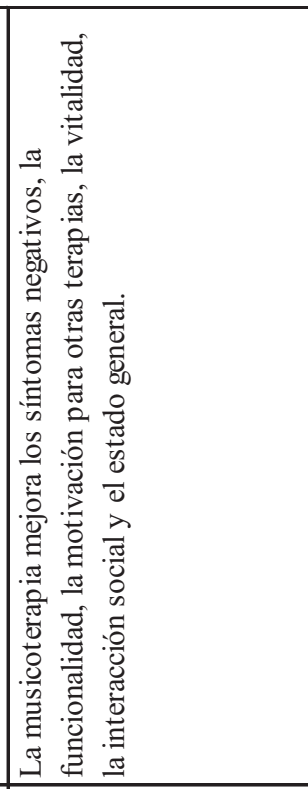 & 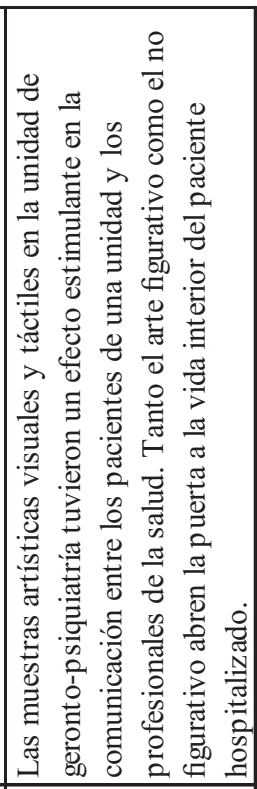 & 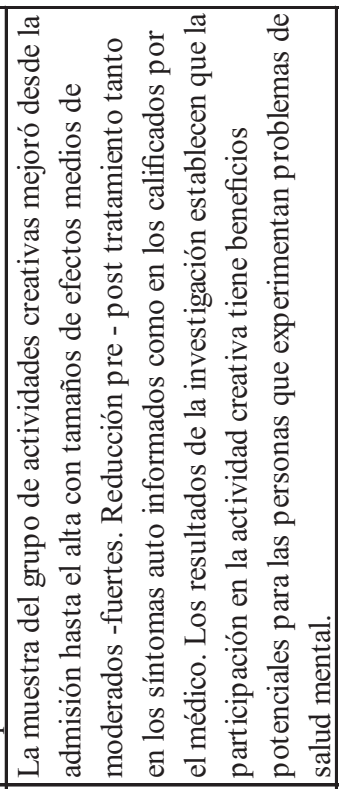 \\
\hline 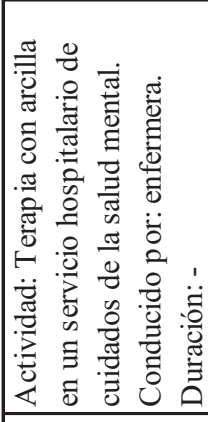 & 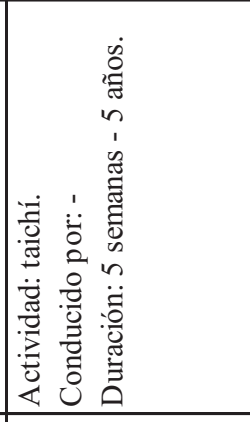 & 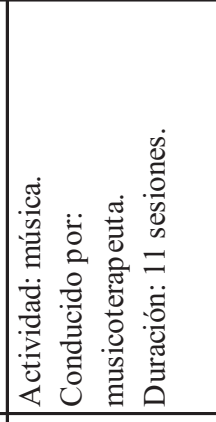 & 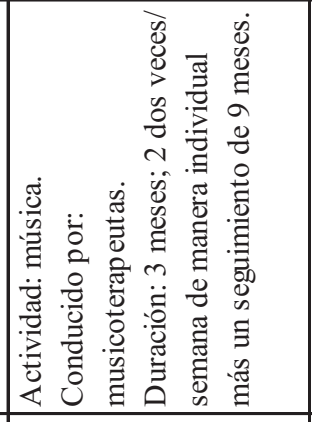 & 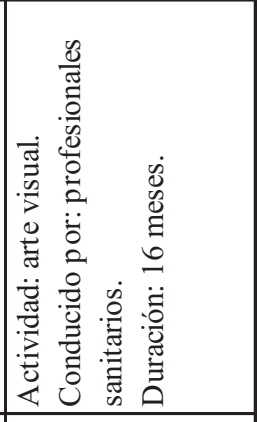 & 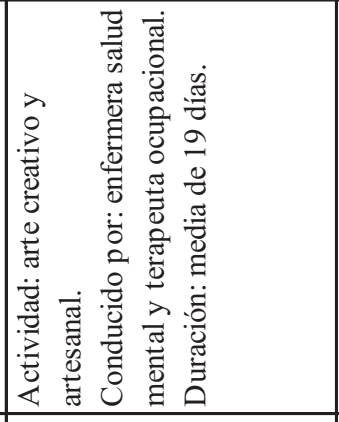 \\
\hline 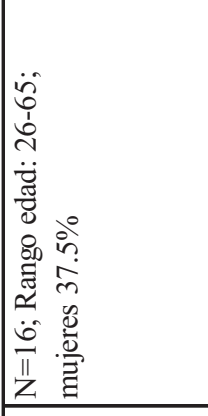 & 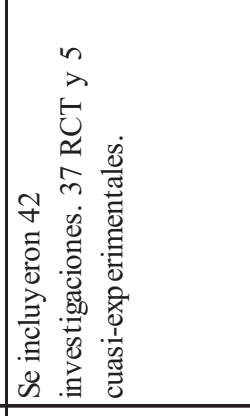 & 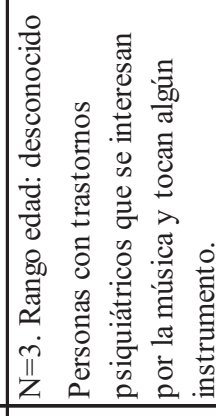 & 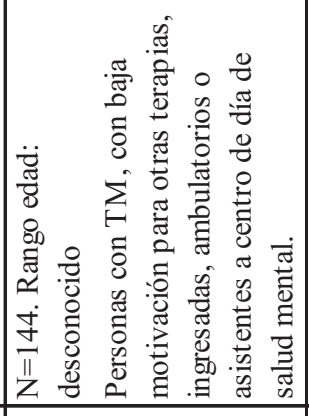 & 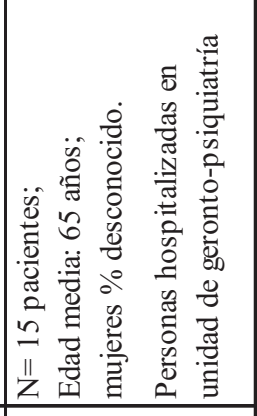 & 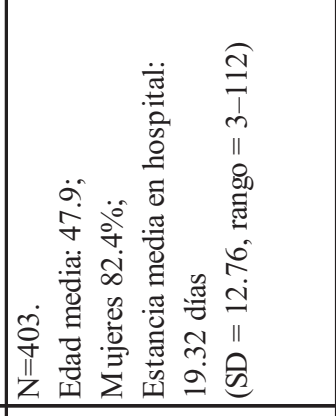 \\
\hline 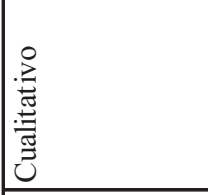 & & 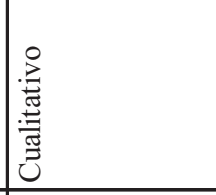 & 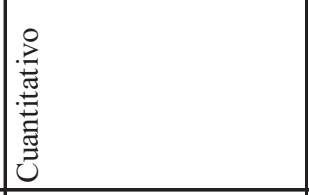 & & : \\
\hline 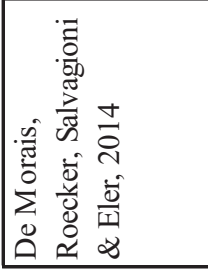 & 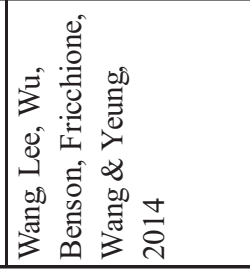 & 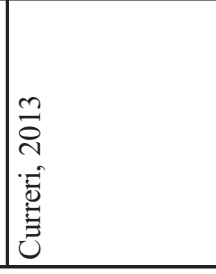 & 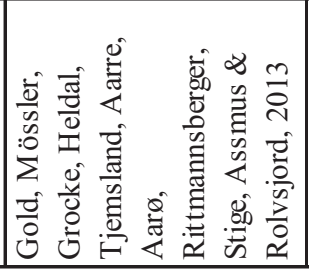 & 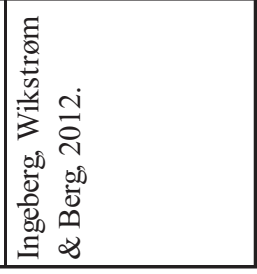 & 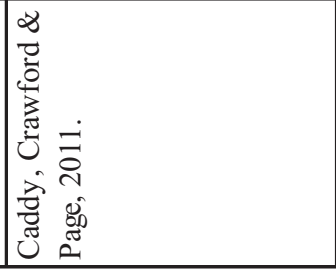 \\
\hline
\end{tabular}




\begin{tabular}{|c|c|c|c|}
\hline 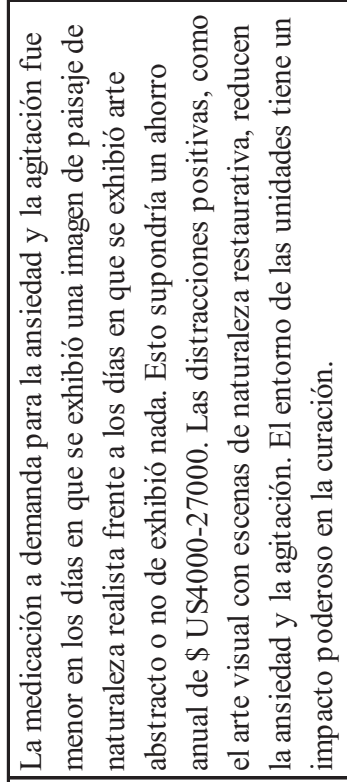 & 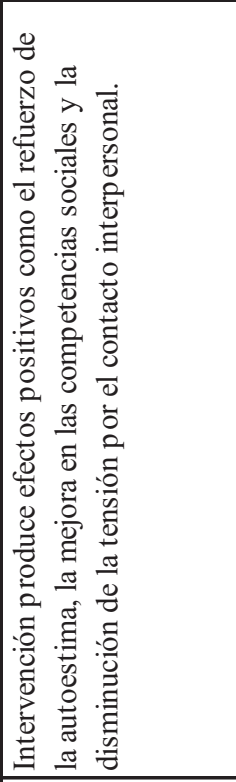 & 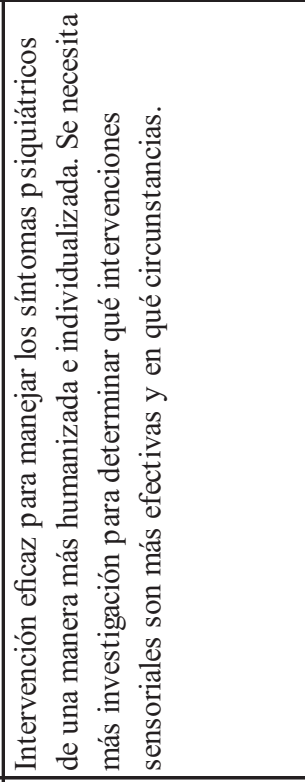 & 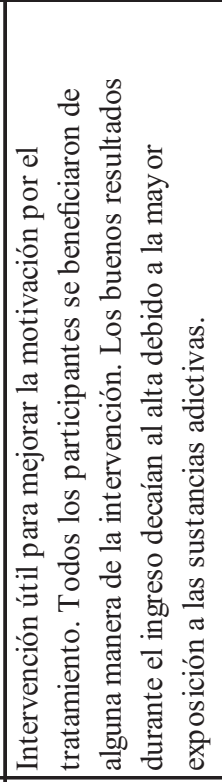 \\
\hline 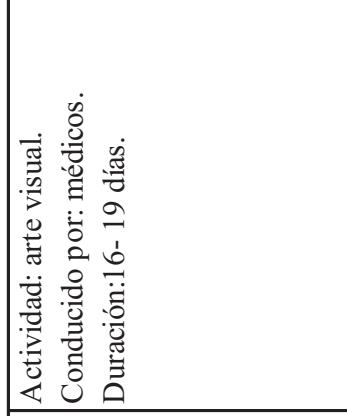 & 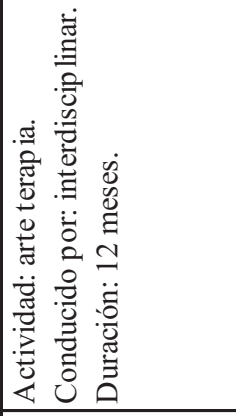 & 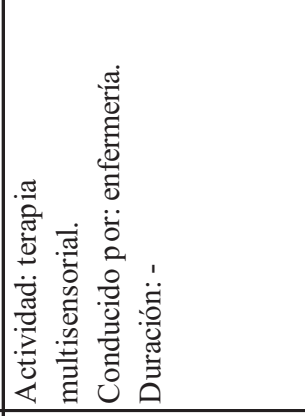 & 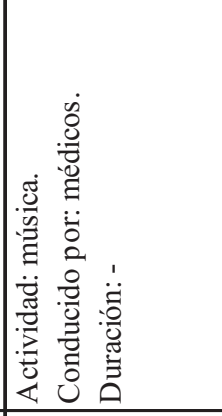 \\
\hline 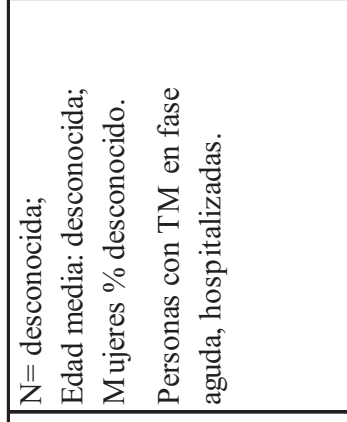 & 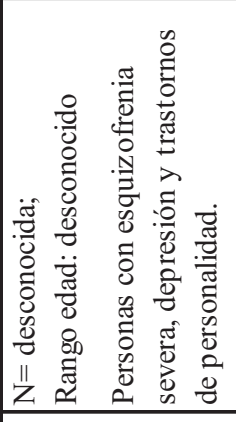 & 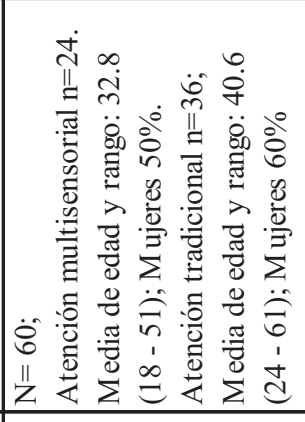 & 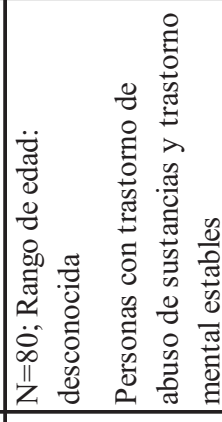 \\
\hline 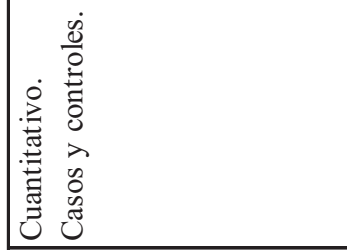 & 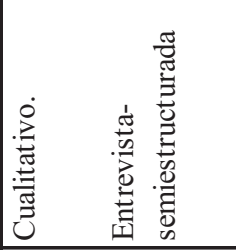 & $\begin{array}{l}\text { o } \\
0 \\
0 \\
0 \\
0 \\
0 \\
\lambda \\
0 \\
0 \\
0 \\
0 \\
0\end{array}$ & 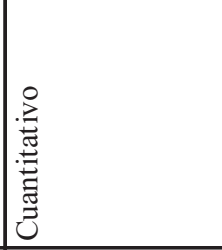 \\
\hline 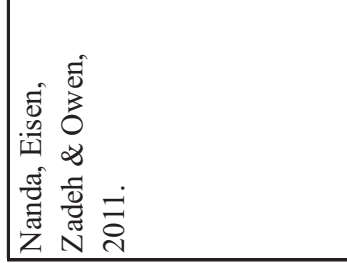 & 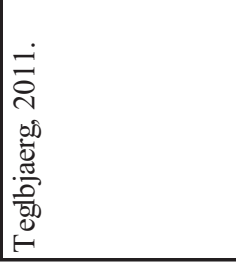 & 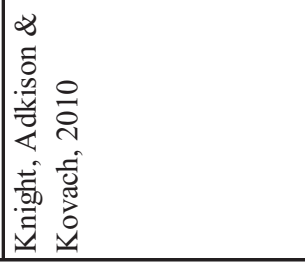 & 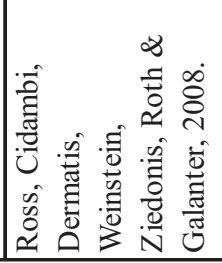 \\
\hline
\end{tabular}

